\title{
Studies on the efficiency of different inoculation methods of rice white-tip nematode, Aphelenchoides besseyi
}

\author{
Jialian XIE $^{1}$, Fang YANG ${ }^{1}$, Yanping WANG ${ }^{1}$, Yunliang PENG ${ }^{1,2, *}$ and Hongli JI ${ }^{1, *}$ \\ ${ }^{1}$ MOA Key Laboratory of Integrated Management of Pests on Crops in Southwest China, Institute of Plant Protection, \\ Sichuan Academy of Agricultural Sciences, Chengdu 610066, P.R. China \\ ${ }^{2}$ China National Rice Research Institute, Hangzhou 310014, P.R. China
}

Received: 14 November 2018; revised: 21 January 2019

Accepted for publication: 21 January 2019; available online: 1 March 2019

\begin{abstract}
Summary - To fulfil different research purposes, five methods to inoculate Aphelenchoides besseyi onto seedlings and panicles of rice, Oryza sativa, were evaluated in this study and the efficiency of the inoculation methods assessed by success rates and recovery rates. Among the three methods leading to the infection of young seedlings without obvious mechanical wounds, i.e., water flotation, seed soaking and leaf spraying, the inoculation of 125 nematodes plant ${ }^{-1}$ by the water flotation method gave rise to the highest success rate and recovery rate, 95.6 and $8.3 \%$, respectively, in growth chamber experiments. When conducted in the plastic house, seed soaking and leaf spraying of 125 nematodes plant ${ }^{-1}$ resulted in 75.6 and $66.7 \%$ success rate, respectively, and 155.7 and $178.1 \%$ recovery rates, respectively. The injection or spraying of 2000 nematodes panicle ${ }^{-1}$ at the booting or flowering stage gave $100 \%$ infection.
\end{abstract}

Keywords - flotation, injection, nematode recovery, Oryza sativa, soaking, spraying, success rate.

Rice (Oryza sativa) is one of the major food crops for more than a third of the world's population. Among over 200 plant-parasitic nematodes associated with rice (Prot, 1994), the rice white-tip nematode, Aphelenchoides besseyi, is considered to be a major contributor to the seed-borne pathogens of rice (Duncan \& Moens, 2013), causing substantial annual losses world-wide of $c a$ US\$ 16 billion (Lilley et al., 2011). It is widely distributed throughout almost all the rice-growing regions of the world but is still a quarantine pest (Fortuner \& Orton Williams, 1975). A reduction in rice yield as severe as 30-70\% has been reported (Tikhonova, 1966; Muthukrishnan et al., 1974; Lin et al., 2004; Tulek \& Cobanoglu, 2010).

As a seed-borne nematode, A. besseyi survives anhydrobiotically in stored grain for several years (Tiwari \& Khare, 2003). When infected seed is planted, the nematodes become active, emerge from the seed and feed on the above-ground tissues of rice, parasitising the leaves and other young tissues as a foliar nematode. During the early growth stages of rice, A. besseyi is found in low numbers in all green tissues, but is more abundant in the stem. A rapid increase in nematode numbers takes place at the flowering stage, when nematodes are mainly found in the florets of the panicles (Huang \& Huang, 1972). This causes typical symptoms of 'white-tip' in leaves, which later become necrotic, then die and disintegrate (Togashi \& Hoshino, 2001; Duncan \& Moens, 2013). Some $O$. sativa subsp. japonica rice varieties do not show the typical white-tip symptoms but manifest smaller grains and erect panicles; other varieties, mainly of $O$. sativa subsp. indica, do not have any obvious symptoms (Liu et al., 2008). Aphelenchoides besseyi is amphimictic and males are usually abundant (Huang et al., 1979); however, parthenogenetic reproduction has also been found (Nandini et al., 2001). It is a facultative parasite and can feed and multiply on fungi in addition to more than 200 types of plants in 35 genera, of which strawberry (Fragaria $\times$ ananassa) is another economically important host (Yu \& Tsay, 2004).

To develop a control strategy of the nematode, it is necessary to study the parasitism and pathogenicity of A. besseyi, the interaction between the nematode and host, the efficiency of nematicides, and the resistance in rice against $A$. besseyi, either in the laboratory or in the field. Therefore, simple and efficient methods to establish successful infection for different purposes are required. At present, the published methods for inoculation include

*Corresponding authors, e-mails: Pengyunliang@aliyun.com; Hongli.Ji@ Jihongli.com 
injecting the sprout using a syringe (McGawley et al., 1984; Jamali et al., 2006), adding nematode suspensions to the soil (Togashi \& Hoshino, 2003; Jamali et al., 2006), leaf spraying, and inoculation of the nematode by inserting a plastic tube at seedling and booting stages (Jamali et al., 2006); however, these methods either cause damage to the host and retain little moisture, or have very low inoculation success rates and are timeconsuming. Here, we established four new inoculation methods including water flotation, seed soaking, panicle injection and panicle spraying. Moreover, we re-evaluated the leaf-spraying method (Jamali et al., 2006).

\section{Materials and methods}

\section{NEMATODE PREPARATION}

Aphelenchoides bessyi was isolated from infected rice seeds of 'AnHuil' (O. sativa subsp. japonica) by soaking seeds in tap water; $72 \mathrm{~h}$ later nematodes were collected and surface-sterilised with $0.1 \%$ streptomycin sulphate for $10 \mathrm{~min}$, washed three times with double-distilled water, and subsequently cultured on carrot discs at $25^{\circ} \mathrm{C}$ for 30 days as described by Tulek et al. (2009). The nematodes were then collected in tap water and adjusted to a different concentration before further application. Clean tap water without any nematodes was used as the blank control in all experiments.

\section{PLANT MATERIAL AND GROWTH CONDITIONS}

Seeds of a japonica rice variety, 'Lijiangxintuan Heigu', were used in all experiments; they were soaked in hot water at $56^{\circ} \mathrm{C}$ for $15 \mathrm{~min}$ to kill the nematodes already in the glumes (Fortuner \& Orton Williams, 1975). The seedlings for growth chamber inoculations were grown in autoclaved sand mixed with $0.2 \%(\mathrm{~m} / \mathrm{v})$ superabsorbent polymer (SAP) (Reversat et al., 1999). The growth chamber was set at $27^{\circ} \mathrm{C}$ under a $16 \mathrm{~h}$ light: $8 \mathrm{~h}$ dark regime and a relative humidity (RH) of $70-80 \%$.

The seedlings for the plastic house evaluations were grown at approximately $26^{\circ} \mathrm{C}$ and $60-70 \% \mathrm{RH}$ in soil plots. Plastic films were used to cover the plants to retain moisture, and the temperature and $\mathrm{RH}$ increased to $28-30^{\circ} \mathrm{C}$ and $70-90 \%$, respectively. Plots were $1.5 \mathrm{~m} \times$ $2.0 \mathrm{~m}$ and independently irrigated, and caged with plastic nets to protect against insects. An earth levee of $25 \mathrm{~cm}$ height was made around each plot to maintain the water level and to prevent the spread of nematodes.

\section{INOCULATION METHODS}

\section{Water flotation}

Hot water-treated seeds were germinated and planted individually into the centre of a $10 \mathrm{ml}$ centrifuge tube containing $4 \mathrm{ml} \mathrm{SAP}$ substrate; the tubes were capped and the plants were grown for 3 days in a growth chamber. Subsequently, $2 \mathrm{ml}$ of nematode suspensions in tap water containing either $0,125,250$ or 500 nematodes were added to each plant to submerge the growth site of the seedling. The tubes were capped for another 5 days to retain moisture. Each inoculum level was conducted on 15 seedlings, and the experiment was independently repeated three times. To evaluate the later effect of inoculation, another 15 seedlings inoculated with 125 nematodes were transplanted into a plastic house plot at 20 days post inoculation (dpi); plants were grown spaced $20 \mathrm{~cm}$ apart in rows $15 \mathrm{~cm}$ apart. Symptoms were observed and recorded 60 days after transplanting.

\section{Seed soaking}

Sixty hot water-treated seeds were soaked in $12 \mathrm{ml}$ nematode suspension in tap water containing either 0 , 125,250 or 500 nematodes seed $^{-1}$ for $24 \mathrm{~h}$. Subsequently, ten seeds were planted in a $8.5 \mathrm{~cm}$ diam. plastic box containing SAP substrate and covered with plastic film for 7 days. Each inoculum level was conducted with six boxes (replicates), and the experiment was independently repeated three times. For plastic house plot inoculation, 15 seeds were soaked in $3 \mathrm{ml}$ suspension containing 1875 nematodes, i.e., 125 nematode seed $^{-1}$. Each seed was then planted in a plastic house plot. The plants were spaced $20 \mathrm{~cm}$ apart in rows $15 \mathrm{~cm}$ apart and covered with plastic membrane for 14 days. Symptoms were observed and recorded 60 days after planting. The experiment was independently repeated three times.

\section{Leaf spraying}

Eight hot water-treated seeds were germinated and planted in a $8.5 \mathrm{~cm}$ diam. plastic box containing SAP substrate, and placed in a growth chamber. When the plants had two leaves, $2 \mathrm{ml}$ nematode suspensions with $0.01 \%$ Tween-20 containing either $0,125,250$ or 500 nematodes plant $^{-1}$ were sprayed evenly onto the rice leaves of plants in each box using a $4 \mathrm{ml}$ perfume sprayer. Plastic film was placed over the plants to retain moisture for 7 days. Each inoculum level was conducted with six boxes (replicates), and the experiment was independently repeated three times. For plastic house plot inoculation, ten nematode-free seeds were planted in a $15 \mathrm{~cm} \times$ 
$20 \mathrm{~cm}$ plot, and 14-day-old seedlings were sprayed with $2 \mathrm{ml}$ nematode suspension with $0.01 \%$ Tween20 containing 125 nematodes plant ${ }^{-1}$ as in the growth chamber experiment. The plants were covered with plastic film for 7 days and then seedlings were transplanted. The plants were spaced $20 \mathrm{~cm}$ apart in rows $15 \mathrm{~cm}$ apart and covered with plastic membrane 14 days after spraying. Symptoms were observed and recorded 60 days after transplanting. The experiment was independently repeated three times.

\section{Panicle injection}

Hot water-treated seeds were planted in a plastic house plot. At the booting stage, $0.2 \mathrm{ml}$ nematode suspension containing 2000 nematodes was injected into the sheath of each flag leaf enclosing the panicle using a $1 \mathrm{ml}$ sterile plastic syringe. Plastic bags with small holes were used to envelop the top part of the inoculated plants to retain moisture for 5 days. Eight similarly sized panicles from a total of eight rice plants were selected and inoculated; $0.2 \mathrm{ml} 0.01 \%$ Tween 20 in nematode-free tap water was used as the control. The experiment was independently repeated three times.

\section{Panicle spraying}

Hot water-treated seeds were planted in a plastic house plot. At the flowering stage, $0.2 \mathrm{ml}$ nematodes suspension containing 2000 nematodes was sprayed onto each panicle with a $2 \mathrm{ml}$ perfume sprayer. Plastic bags with small holes were used to envelop the panicles to retain moisture for 5 days. Eight similarly sized panicles from a total of eight rice plants were selected and sprayed; $0.01 \%$ Tween-20 in nematode-free tap water was used as the control. The experiment was independently repeated three times.

\section{DATA COLLECTION AND ANALYSIS}

For growth chamber inoculation, the above-ground part of seedlings at 25 dpi was cut into approximately $1 \mathrm{~cm}$ long pieces, immersed in $0.01 \%$ Tween-20 and placed on a shaker at $40 \mathrm{rpm}$ for $24 \mathrm{~h}$. Then the suspensions and rinsing water were collected together, viewed under a stereomicroscope (Leica S8AP0; Leica Microsystems) and the number of nematodes was recorded.

For plastic house inoculation, in seed soaking and leaf spraying methods, all the ripe seeds from individual inoculated plants were collected; in panicle injection and panicle spraying methods, ripe seeds were collected from individual inoculated panicles only. The collected seeds were gently ground to separate glumes and grains, then the glumes and grains were soaked in tap water on a shaker at $40 \mathrm{rpm}$ for $8 \mathrm{~h}$. The suspensions and rinsing water were collected together, and the numbers of nematodes from seeds of each plant or panicle were counted as above.

Plants were considered to be infected if there were nematodes recovered from the seedlings or the harvested seeds. The efficiency of the different methods was evaluated by the success rate and recovery rate. The success rate was defined as the percentage of infected plants out of the inoculated plants, i.e., success rate $(\%)=100 \times$ number of infected plants (or panicles)/number of inoculated plants (or panicles). The recovery rate was defined as the rate between average number of nematodes recovered from the infected plants and the number of inoculated nematodes per plants, i.e., recovery rate $(\%)=100 \times$ average number of the nematodes recovered from infected plants (or panicles)/number of nematodes inoculated per plant (or panicle).

Statistical analysis was conducted using software $\mathrm{R}$ (version 3.3.0) (R Core Team, 2016). Comparisons of differences between inoculations were performed by the Mann-Whitney test, followed by $P$ value adjustment with the method of less conservative corrections $(\alpha=0.05)$ of Holm (1979).

\section{Results}

\section{INOCULATION CONDUCTED IN A GROWTH CHAMBER}

For the water flotation method, mean inoculation success rates with the inocula at 125, 250 and 500 nematodes plant $^{-1}$ were $95.6,95.6$ and $97.8 \%$, respectively, and there were no significant differences between different inocula (Table 1). The recovery rates of this method with the inocula at 125,250 and 500 plant $^{-1}$ were $8.3,5.3$ and $1.6 \%$, respectively, and the inoculum at 125 nematodes plant ${ }^{-1}$ led to a significantly higher recovery than the other two (Table 1). After transplanting into the plastic house plot, the seedling of 'Lijiangxintuan Heigu' inoculated by water flotation at 125 nematodes plant ${ }^{-1}$ gave rise to the typical white-tip symptoms. The percentage of plants showing symptoms was $62.2 \%$.

For the seed-soaking method, the mean inoculation success rates with the inocula at 125, 250 and 500 seed $^{-1}$ were $48.9,36.7$ and $50.0 \%$, respectively (Table 1), and the success rate with the inoculum at 250 nematodes seed $^{-1}$ was significantly lower than that with the other two inocula. The recovery rates of this method with the inocula at 125, 250 and 500 seed $^{-1}$ were 6.6, 3.8 and $1.4 \%$, respectively; the inoculum at 125 nematodes seed ${ }^{-1}$ 
J. Xie et al.

Table 1. Inoculation efficiency of rice, Oryza sativa, by Aphelenchoides besseyi with different methods conducted in growth chambers.

\begin{tabular}{|c|c|c|c|c|}
\hline Method & Inoculum plant $^{-1}$ & Success rate $(\%)$ & Recovery rate $(\%)$ & $\begin{array}{l}\text { Number of recovered nematodes } \\
\text { from infected plants }\end{array}$ \\
\hline Water floatation $(\mathrm{n}=45)$ & $\begin{array}{l}500 \\
250 \\
125\end{array}$ & $\begin{array}{l}97.8 \pm 3.1 \mathrm{a} \\
95.6 \pm 3.1 \mathrm{a} \\
95.6 \pm 3.1 \mathrm{a}\end{array}$ & $\begin{array}{l}1.6 \pm 0.1 \mathrm{a} \\
5.3 \pm 1.6 \mathrm{~b} \\
8.3 \pm 3.0 \mathrm{c}\end{array}$ & $\begin{array}{c}8.0 \pm 0.1 \mathrm{a} \\
13.2 \pm 2.3 \mathrm{~b} \\
10.4 \pm 2.2 \mathrm{c}\end{array}$ \\
\hline Seed soaking $(\mathrm{n}=18)$ & $\begin{array}{l}500 \\
250 \\
125\end{array}$ & $\begin{array}{l}50.0 \pm 8.3 b \\
36.7 \pm 7.6 c \\
48.9 \pm 6.1 b\end{array}$ & $\begin{array}{l}1.4 \pm 0.2 \mathrm{a} \\
3.8 \pm 0.2 \mathrm{~d} \\
6.6 \pm 0.2 \mathrm{e}\end{array}$ & $\begin{array}{l}7.2 \pm 3.2 \mathrm{a} \\
9.6 \pm 2.7 \mathrm{c} \\
8.3 \pm 1.9 \mathrm{a}\end{array}$ \\
\hline Leaf spraying $(\mathrm{n}=18)$ & $\begin{array}{l}500 \\
250 \\
125\end{array}$ & $\begin{array}{c}44.5 \pm 9.7 b \\
54.9 \pm 11.8 b \\
45.1 \pm 1.0 b\end{array}$ & $\begin{array}{l}1.1 \pm 0.2 \mathrm{a} \\
1.9 \pm 0.3 \mathrm{f} \\
4.6 \pm 0.8 \mathrm{~b}\end{array}$ & $\begin{array}{l}5.5 \pm 1.4 \mathrm{~d} \\
4.9 \pm 0.2 \mathrm{~d} \\
5.7 \pm 1.9 \mathrm{~d}\end{array}$ \\
\hline
\end{tabular}

Success rate $(\%)=100 \times($ infected plants/inoculated plants); recovery rate $(\%)=100 \times$ (average number of nematodes recovered from infected plants/inoculated nematodes per plant). In water-flotation, seed-soaking and leaf-spraying experiments, 15, 60 and 60 seedlings, respectively, were inoculated and investigated at each inoculum amount; data are means \pm standard error based on all replicates of three experimental repeats. Different letters in the same column indicate significant difference based on the Mann-Whitney test ( $\alpha=0.05)$.

led to a significantly higher recovery in comparison to other inocula (Table 1).

For the leaf-spraying method in the growth chamber, mean inoculation success rates with inocula at 125, 250 and 500 nematodes plant $^{-1}$ were $45.1,54.9$ and $44.5 \%$, respectively (Table 1), and there were no significant differences between the different inocula. The recovery rates of this method were $4.6,1.9$ and $1.1 \%$ at the above inoculum levels, respectively; the recovery resulting from the spraying of 125 nematodes plant ${ }^{-1}$ was significantly higher than that of other two inocula.

\section{INOCULATION CONDUCTED IN A PLASTICHOUSE}

To check if the seed-soaking and leaf-spraying methods could be used on a large scale, the methods with the inoculum at 125 nematodes seed ${ }^{-1}$ or seedling ${ }^{-1}$ were used in plastic house experiments. The success rates of the two methods were 75.6 and $66.7 \%$, respectively, and the respective recovery rates were 155.7 and $178.1 \%$ (Table 2). The percentages of plants showing whitetip symptoms at later stages of growth were 55.6 and $51.1 \%$ from the seed-soaking and leaf-spraying methods, respectively.

The success rates of the inoculation methods by panicle spraying and panicle injection were both $100 \%$ (Table 3). The mean number of recovered nematodes from the sprayed panicles was 101.83 panicle $^{-1}$, while the panicle injection method gave rise to a significantly lower mean number at 53.00 panicle $^{-1}$ after the recovery test (Table 3).

\section{Discussion}

The five inoculation methods, water flotation, seed soaking, leaf spraying, panicle injection and panicle spraying, all resulted in the establishment of infection of rice by $A$. besseyi; the water flotation method was the most efficient indoor method in both terms of rates of infected plants and recovered nematodes from the infected plants. Using this method, nearly all the inoculated seedlings were infected by nematodes and this led to the typical white-tip symptoms at later growth stages of the plants. Such a method could be ideal for laboratory studies of early interactions between $A$. besseyi and rice. In our preliminary test, a lower number of nematodes in the inoculum failed to increase, and even decreased, the success rate and nematode recovery (data not shown); this may be because the establishment of infection needs a minimum number of nematodes. Inoculating nematode suspensions into the soil has also been reported to establish infection (Togashi \& Hoshino, 2003; Jamali et al., 2006). However, similar to the method of soil inoculation, the application of nematode suspension to SAP substrate has repeatedly led to an overall low rate of infected plants ranging from 30 to $40 \%$, even when the inoculum was as high as 1000 nematode seed ${ }^{-1}$ (Table S1). The possible reason for such low infection could be the difficulty for nematodes to access seeds in the soil or the SAP substrate after the dilution of the inoculum in the substrates. To establish the water flotation method, we used SAP in a $10 \mathrm{ml}$ centrifuge tube, which concentrated the nematodes within a small area. This would in part improve inoculation efficiency. 
Table 2. Inoculation efficiency of rice, Oryza sativa, by seed soaking and leaf spraying of a suspension of Aphelenchoides besseyi in glasshouse conditions.

\begin{tabular}{lccccc}
\hline Method & Inoculum plant $^{-1}$ & Success rate (\%) & Recovery rate (\%) & $\begin{array}{c}\text { Number of recovered nematodes } \\
\text { from infected plants }\end{array}$ & $\begin{array}{c}\text { Rate of white-tip } \\
\text { symptoms }(\%)\end{array}$ \\
\hline Seed soaking & 125 & $75.6 \pm 3.1 \mathrm{a}$ & $155.7 \pm 38.9 \mathrm{a}$ & $194.7 \pm 54.5 \mathrm{a}$ & $55.6 \pm 3.1 \mathrm{a}$ \\
Leaf spraying & 125 & $66.7 \pm 5.4 \mathrm{~b}$ & $178.1 \pm 24.4 \mathrm{a}$ & $222.6 \pm 36.9 \mathrm{~b}$ & $51.1 \pm 6.3 \mathrm{a}$ \\
\hline
\end{tabular}

For definition of success rate and recovery rate see Table 1 . In seed-soaking and leaf-spraying experiment,s 15 seedlings were inoculated and investigated; data are means \pm standard error $(n=45)$ based on all replicates of three experimental repeats. Different letters in the same column indicated a significant difference based on Mann-Whitney test $(\alpha=0.05)$.

Table 3. Inoculation efficiency of rice, Oryza sativa, panicles by panicle injection and panicle spraying of a suspension of Aphelenchoides besseyi in plastic-house conditions.

\begin{tabular}{lcccc}
\hline Method & Inoculum panicle ${ }^{-1}$ & Success rate (\%) & Recovery rate $(\%)$ & $\begin{array}{c}\text { Nematodes recovered } \\
\text { from each panicle }\end{array}$ \\
\hline Panicle injection & 2000 & $100.0 \pm 0.0 \mathrm{a}$ & $2.7 \pm 0.3 \mathrm{a}$ & $53.0 \pm 5.2 \mathrm{a}$ \\
Panicle spraying & 2000 & $100.0 \pm 0.0 \mathrm{a}$ & $5.1 \pm 1.2 \mathrm{~b}$ & $101.8 \pm 23.8 \mathrm{~b}$ \\
\hline
\end{tabular}

For definition of success rate and recovery rate see Table 1. Eight plants were inoculated on one panicle at 2000 nematodes plant $^{-1}$; data are means \pm standard error $(n=24)$ based on all replicates of three experimental repeats. Different letters in the same column indicated a significant difference based on Mann-Whitney test $(\alpha=0.05)$.

Another benefit of this method is that it could synchronise infection at a certain time after inoculation, which could be used in experiments for observation of infection development, for example.

The success rates of the seed-soaking method and the leaf-spraying method in growth chamber conditions were generally between 40 and $50 \%$, but in the plastic house plot values they were 67 and $76 \%$, respectively. This indicated lower levels of infection rates due to limitations in the recovery test, as well as the possibility of a second infection occurring in the transplanted seedlings. Typical white-tip symptoms were observed in successfully infected plants in the plastic house and the percentage of plants showing symptoms was over 50\%, which was consistent with the result of Jamali et al. (2006). These two methods were convenient to conduct, and could be used for experiments such as resistance screening, which requires inoculation of a large number of plants.

In addition, it is often necessary to prepare a large quantity of nematode-infected seeds for in vivo test of nematicides. Using the panicle-injection and paniclespraying methods developed in this study, it is possible to obtain a large quantity of infected seeds. The methods also make it possible to keep a bank of $A$. besseyi at low cost by keeping the nematodes in anhydrobiosis in dry seeds.

\section{Acknowledgements}

The authors acknowledge the financial support of the National Natural Science Foundation of China (Grant No. 31701769), Special Grants from China Agriculture Research System (CARS-01-38) and Research foundation for Ph.D. of the Sichuan Academy of Agricultural Sciences (2016QNJJ).

\section{References}

Duncan, L.W. \& Moens, M. (2013). Migratory endoparasitic nematodes. In: Perry, R.N. \& Moens, M. (Eds). Plant nematology, 2nd edition. Wallingford, UK, CAB International, pp. 144-178.

Fortuner, R. \& Orton Williams, K.J. (1975). Review of the literature on Aphelenchoides besseyi Christie, 1942, the nematode causing "white tip" disease in rice. Helminthological Abstracts, Series B: Plant Nematology 44, 1-40.

Holm, S. (1979). A simple sequentially rejective multiple test procedure. Scandinavian Journal of Statistics 6, 65-70. https://www.jstor.org/stable/4615733.

Huang, C.S. \& Huang, S.P. (1972). Bionomics of white-tip nematode, Aphelenchoides besseyi in rice florets and developing grains. Botanical Bulletin of Academia Sinica 13, 1-10.

Huang, C.S., Huang, S.P. \& Chiang, Y.C. (1979). Mode of reproduction and sex ratio of rice white-tip nematode, Aphelen- 
choides besseyi. Nematologica 25, 255-260. DOI: 10.1163/ 187529279x00271

Jamali, S., Pourjam, E., Alizadeh, A. \& Alinia, F. (2006). Evaluation of different inoculation methods of rice whitetip nematode, Aphelenchoides besseyi, in pathogenicity test. Iranian Journal of Plant Pathology 42, 193-195. [Abstr.]

Lilley, C.J., Kyndt, T. \& Gheysen, G. (2011). Nematode resistant GM crops in industrialised and developing countries. In: Jones, J.T., Gheysen, G. \& Fenoll, C. (Eds). Genomics and molecular genetics of plant-nematode interactions. Heidelberg, Germany, Springer, pp. 517-541.

Lin, M., Ding, X., Wang, Z., Zhou, F. \& Lin, N. (2004). Description of Aphelenchoides besseyi from abnormal rice with 'small grains and erect panicles' symptom in China. Rice Science 12, 289-294.

Liu, W.H., Lin, M.S., Li, H.M. \& Sun, M.J. (2008). Dynamic development of Aphelenchoides besseyi on rice plant by artificial inoculation in the greenhouse. Agricultural Sciences in China 7, 970-976. DOI: 10.1016/s1671-2927(08)60136-4

McGawley, E.C., Rush, M.C. \& Hollis, J.P. (1984). Occurrence of Aphelenchoides besseyi in Louisiana rice seed and its interaction with Sclerotium oryzae in selected cultivars. Journal of Nematology 16, 65-67.

Muthukrishnan, T.S., Rajendran, G. \& Chandrasekaran, J. (1974). Studies on the white-tip nematode of rice, Aphelenchoides besseyi in Tamil Nadu. Indian Journal of Nematology 4, 188-193.

Nandini, G.N., Mathur, V.K., Ramasundaram, P. \& Sabesh, M. (2001). Reproductive variations in Aphelenchoides besseyi populations. Indian Journal of Nematology 31, 115-119.

Prot, J.C. (1994). Combination of nematodes, Sesbania rostrata, and rice: the two sides of the coin. International Rice Research Notes 19, 30-31.
$\mathrm{R}$ Core Team (2016). $R$ foundation for statistical computing. Vienna, Austria, R Foundation for Statistical Computing.

Reversat, G., Boyer, J., Sannier, C. \& Pando-Bahuon, A. (1999). Use of a mixture of sand and water-absorbent synthetic polymer as substrate for the xenic culturing of plant-parasitic nematodes in the laboratory. Nematology 1, 209-212. DOI: $10.1163 / 156854199508027$

Tikhonova, L.V. (1966). [Aphelenchoides besseyi Christie, 1942 (Nematoda, Aphelenchoididae) on rice and method of control.] Zoologicheskii Zhurnal 45, 1759-1766.

Tiwari, S.P. \& Khare, M.N. (2003). White tip caused by Aphelenchoides besseyi, an important seed-borne disease of rice. In: Trivedi, P.C. (Ed.). Advances in nematology. Jodhpur, India, Scientific Publishers (India), pp. 103-114.

Togashi, K. \& Hoshino, S. (2001). Distribution pattern and mortality of the white tip nematode, Aphelenchoides besseyi (Nematoda: Aphelenchoididae), among rice seeds. Nematology 3, 17-24. DOI: 10.1163/156854101300106847

Togashi, K. \& Hoshino, S. (2003). Trade-off between dispersal and reproduction of a seed-borne nematode, Aphelenchoides besseyi, parasitic on rice plants. Nematology 5, 821-829. DOI: $10.1163 / 156854103773040727$

Tulek, A. \& Cobanoglu, S. (2010). Distibution of the rice white tip nematode, Aphelenchoides besseyi, in rice growing areas in the Thrace region of Turkey. Nematologia Mediterranea 38, 215-217.

Tulek, A., Kepenekci, I., Cobanoglu, S., Hekimhan, H., Devran, Z., Melik, B. \& Elekcioglu, H.I. (2009). A new culturing method for the rice white tip nematode, Aphelenchoides besseyi Christie, 1942, on carrot discs. Russian Journal of Nematology 17, 143-144.

Yu, P.C. \& Tsay, T.T. (2004). Occurrence of a foliar nematode disease of fern in Taiwan. Plant Pathology Bulletin 13, 35-44.

Table S1. Inoculation efficiency of rice, Oryza sativa, by Aphelenchoides besseyi with substrate infestation method.

\begin{tabular}{|c|c|c|c|}
\hline Inoculum plant ${ }^{-1}$ & Success rate $(\%)$ & Recovery rate $(\%)$ & $\begin{array}{l}\text { Number of recovered nematodes } \\
\text { from infected plants }\end{array}$ \\
\hline 1000 & $38.9 \pm 3.8 \mathrm{a}$ & $0.4 \pm 0.0 \mathrm{a}$ & $4.4 \pm 0.3 \mathrm{a}$ \\
\hline 500 & $37.8 \pm 6.9 \mathrm{a}$ & $0.6 \pm 0.1 \mathrm{a}$ & $3.3 \pm 0.9 \mathrm{ab}$ \\
\hline 250 & $33.3 \pm 6.6 a$ & $0.9 \pm 0.01 b$ & $2.2 \pm 0.3 c$ \\
\hline 125 & $41.1 \pm 3.8 \mathrm{a}$ & $2.0 \pm 0.2 \mathrm{~d}$ & $2.5 \pm 0.3 c$ \\
\hline
\end{tabular}

Ten seeds were planted in $200 \mathrm{ml}$ superabsorbent polymer substrate in an $8.5 \mathrm{~cm}$ diam. plastic box, infested by pouring $5 \mathrm{ml}$ nematode suspensions concentrated at either $0,125,250,500$ or 1000 nematodes plant ${ }^{-1}$. For definition of success rate and recovery rate see Table 1. Data are means \pm standard error $(n=9)$ based on all replicates of three experimental repeats. Different letters in the same column indicated a significant difference based on Mann-Whitney test $(\alpha=0.05)$. 